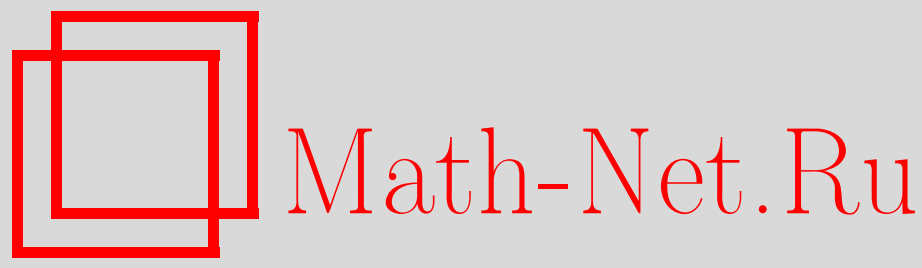

Ю. Е. Кузовлев, Молекулярное случайное блуждание и группа инвариантности уравнения Боголюбова, ТМФ, 2009, том 160, номер 3, 517-533

DOI: https://doi.org/10.4213/tmf6413

Использование Общероссийского математического портала Math-Net.Ru подразумевает, что вы прочитали и согласны с пользовательским соглашением http://www.mathnet.ru/rus/agreement

Параметры загрузки:

IP : 34.229 .108 .108

26 апреля 2023 г., $17: 25: 24$

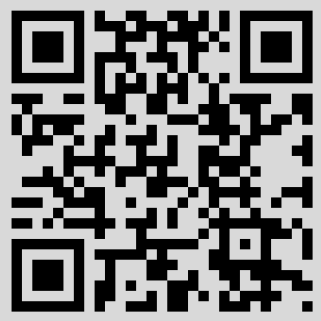




\title{
МОЛЕКУЛЯРНОЕ СЛУЧАЙНОЕ БЛУЖДАНИЕ И ГРУППА ИНВАРИАНТНОСТИ УРАВНЕНИЯ БОГОЛЮБОВА
}

\begin{abstract}
Статистика молекулярного случайного блуждания в газе или жидкости рассмотрена с помощью уравнения Боголюбова для производящего функционала функций распределения. Найдена группа инвариантности этого уравнения и его решений как функций плотности среды. Она индуцирует ряд точных соотношений между вероятностным распределением перемещения блуждающей пробной частицы и ее корреляциями с окружением и, как следствие, налагает серьезные ограничения на возможный вид распределения перемещения. В частности, определенно запрещена (даже для газа Больцмана-Грэда) гауссова асимптотика распределения, но разрешена диффузионная асимптотика со степенными хвостами (обрезанными баллистическим пробегом).
\end{abstract}

Ключевые слова: уравнения Боголюбова-Борна-Грина-Кирквуда-Ивона, производящий функционал Боголюбова, молекулярное случайное блуждание, диффузия, кинетическая теория газов и жидкостей.

\section{1. ВВЕДЕНИЕ}

Беспорядочное блуждание частиц материи - это механизм диффузии и других процессов переноса, а также источник разнообразных шумов и флуктуаций. Какой может быть его статистика? Этот важный вопрос никогда не ставился в рамках строгой статистической механики. Казалось бы, ответ и так очевиден: если уж в газе Лоренца статистика блуждания асимптотически гауссова [1], то тем более это должно быть верно для обычного газа или жидкости. Однако подобное рассуждение теряет убедительность, если заметить, что в нем хаотичность блуждания в смысле гамильтоновой динамики неявно отождествляется со случайностью в смысле теории вероятностей. Первая, как известно, характеризуется "перемешиванием" (или "размешиванием") фазовых траекторий системы [2], тогда как вторая - "статистической независимостью" (или просто "независимостью") их составных частей [3]. Как показал Крылов [2], в общем случае первая не влечет за собой вторую. Поэтому вопрос остается в силе.

* Донецкий физико-технический институт им. А. А. Галкина НАНУ, Донецк, Украина. E-mail: kuzovlev@kinetic.ac.donetsk.ua 
Пояснить этот вопрос можно на примере $N$ твердых шаров в ящике или на торе, трактуя их движение как движение единственного шара в $3 N$-мерном бильярде [4], образованном $N(N-1) / 2$ выпуклыми рассеивателями и напоминающем газ Лоренца. Насколько далеко нужно разнести по времени фрагменты траектории данного шара для того, чтобы они вели себя как статистически независимые? По-видимому, не меньше, чем на характерное время, необходимое шару для того, чтобы узнать о существовании всех рассеивателей и тем самым об устройстве бильярда. Выразим это время через время свободного пробега в исходной трехмерной системе, $\tau \sim\left(\pi r_{0}^{2} \nu v_{0}\right)^{-1}$, где $r_{0}$ - диаметр шаров, $v_{0}-$ их типичная скорость, $\nu=N / \Omega$ - их средняя концентрация, $\Omega$ - объем исходной системы. Поскольку частота столкновений с рассеивателями в $3 N$-мерном бильярде равна суммарной частоте столкновений между произвольными шарами в трехмерной системе, т.е. приблизительно $N / 2 \tau$, то для указанного характерного времени имеем оценку $[N(N-1) / 2] /[N / 2 \tau] \sim N \tau \sim \Omega / \pi r_{0}^{2} v_{0}$. Даже для одного кубического сантиметра воздуха это величина порядка тысячи лет!

Тогда рассчитывать на эргодическое поведение системы $N$ частиц можно лишь применительно к интервалам времени $t \gg N \tau$. С точки зрения физических (пусть даже замкнутых) систем многих частиц это недостижимо большое время. Реально же наблюдению доступны лишь более или менее специфические отрезки фазовой траектории. Как справедливо замечено в книге [5], значимость эргодичности для физики сильно преувеличена, поскольку в реальности предел $N \rightarrow \infty$ предшествует пределу $t / \tau \rightarrow \infty$.

$\mathrm{K}$ таким же выводам подталкивает тот факт, что траектория частицы обычного газа определяется не шестью, как в газе Лоренца, а всеми $6 N$ начальными условиями. Поэтому она проявляет свою индивидуальность (и может быть идентифицирована) лишь после $N$ свободных пробегов и столкновений, для чего требуется время $\sim N \tau$. Соответственно, для того чтобы обнаружить ее эргодические свойства и увидеть, как она разбивается на статистически независимые части, нужны масштабы времени $t \gg N \tau$.

Говоря конкретнее, неэргодичность системы (бесконечно) большого числа частиц означает, что интенсивности наблюдаемых в ней релаксационных и флуктуационных процессов различны на разных фазовых траекториях, как и на разных участках одной и той же трактории [6]. Например, имеются низкочастотные флуктуации индивидуального коэффициента диффузии той или иной частицы [7], выражением которых является существенное отличие вероятностного распределения перемещения частицы от нормального (гауссова) распределения.

Теоретические инструменты для строгого изучения подобной статистики давно созданы Боголюбовым [8] и его последователями. Это цепочка уравнений Боголюбова-Борна-Грина-Кирквуда-Ивона (ББГКИ) и эквивалентное уравнение Боголюбова для производящего функционала многочастичных функций распределения. Однако никто не научился пользоваться ими не обрывая цепочку с помощью "очевидных" гипотез про статистическую независимость, заимствованных из теории вероятностей. Поэтому нужны методы, которые более уважительно относились бы к исходным уравнениям. 
Такой потребностью и вызвана настоящая работа. Мы найдем группу инвариантности уравнения Боголюбова и его решений применительно к задаче о блуждании пробной частицы в термодинамически равновесных газе или жидкости. Затем обсудим следствия точных соотношений этой группы. Продемонстрируем, что они указывают на существенно негауссов характер крупномасштабной асимптотики блуждания, причем, что важно, в случае газа форма асимптотики не зависит (как и введенный выше характерный временной масштаб $N \tau$ ) от плотности газа, даже в пределе Больцмана-Грэда.

\section{2. УРАВНЕНИЯ МОЛЕКУЛЯРНОГО СЛУЧАЙНОГО БЛУЖДАНИЯ}

Пусть ящик объема $\Omega$ содержит $N \gg 1$ одинаковых атомов и еще одну пробную частицу. Атомы имеют массу $m$, координаты $\mathbf{r}_{j}$ и импульсы $\mathbf{p}_{j}, j=1,2, \ldots, N$, и взаимодействуют посредством потенциала $\Phi_{a}\left(\mathbf{r}_{j}-\mathbf{r}_{k}\right)$. Пробная частица имеет массу $M$, координату $\mathbf{R}$, импульс $\mathbf{P}$, ее взаимодействие с атомами описывается потенциалом $\Phi_{b}\left(\mathbf{r}_{j}-\mathbf{R}\right)$. Потенциалы являются сферически-симметричными и короткодействующими с непроницаемой сердцевиной. Вследствие взаимодействий пробная частица совершает хаотическое блуждание, поэтому назовем ее броуновской частищей (БЧ).

Нас интересует вероятностное распределение текущей позиции БЧ $\mathbf{R}(t)$ при условии, что в начальный момент времени $t=0$ она занимала точно известное положение, $\mathbf{R}(0)=\mathbf{R}_{0}$, тогда как персональные позиции атомов все время неизвестны. Простейший статистический ансамбль, отвечающий этому требованию, определяется уравнением Лиувилля $\partial D_{N} / \partial t=\left[H_{N}, D_{N}\right]$ для полной нормированной функции распределения системы $D_{N}$ и начальным условием

$$
\left.D_{N}(t)\right|_{t=0}=\frac{\delta\left(\mathbf{R}-\mathbf{R}_{0}\right) e^{-H_{N} / T}}{\int d \mathbf{R} \int d \mathbf{P} \int_{1} \cdots \int_{N} \delta\left(\mathbf{R}-\mathbf{R}_{0}\right) e^{-H_{N} / T}} .
$$

Здесь $H_{N}$ - полный гамильтониан системы (включающий взаимодействие частиц со стенками ящика) и введено обозначение $\int_{k} \ldots=\iint \ldots d \mathbf{r}_{k} d \mathbf{p}_{k}$. Очевидно, данный ансамбль отличается от канонического ансамбля Гиббса только начальной локализацией БЧ. Последняя не мешает ввести частичные функции распределения (ФР)

$$
F_{n}(t)=\Omega^{n} \int_{n+1} \ldots \int_{N} D_{N}(t)
$$

и затем перейти к термодинамическому пределу $(N \rightarrow \infty, \Omega \rightarrow \infty, \nu=N / \Omega=$ const $)$ точно так же, как это сделано в работе [8]. Перечисляя все аргументы ФР, напишем $F_{n}(t)=F_{n}\left(t, \mathbf{R}, \mathbf{r}^{(n)}, \mathbf{P}, \mathbf{p}^{(n)} \mid \mathbf{R}_{0} ; \nu\right)$, где $\mathbf{r}^{(n)}=\left\{\mathbf{r}_{1}, \ldots, \mathbf{r}_{n}\right\}, \mathbf{p}^{(n)}=\left\{\mathbf{p}_{1}, \ldots, \mathbf{p}_{n}\right\}$. По определению $Ф$ Р ненормированны по отношению к координатам атомов. Вместо этого они должны подчиняться условиям ослабления корреляций при раздвижении частиц, т.е. удовлетворять кластерному свойству (по сути, условию существования термодинамического предела [8], [9]). В нашем контексте, с учетом полной симметрии ФР по отношению к атомам, такие условия можно записать как $F_{n} \rightarrow F_{n-1} G_{m}\left(\mathbf{p}_{n}\right)$ при $\mathbf{r}_{n} \rightarrow \infty$, где $G_{m}(\mathbf{p})=(2 \pi T m)^{-3 / 2} e^{-\mathbf{p}^{2} / 2 T m}-$ максвелловское распределение импульса частицы с массой $m$. Единственное, непринципиальное, 
отличие от рассмотрения в монографии [8] заключается в том, что нумерация ФР начинается с нуля, так что $F_{0}\left(t, \mathbf{R}, \mathbf{P} \mid \mathbf{R}_{0} ; \nu\right)$ описывает БЧ саму по себе, и по отношению к координате БЧ все $Ф$ Р нормированны. В частности, $\int F_{0} d \mathbf{R}=G_{M}(\mathbf{P})$. Исходное уравнение Лиувилля стандартным образом порождает уравнения ББГКИ

$$
\begin{aligned}
\frac{\partial F_{n}}{\partial t}= & {\left[H_{n}, F_{n}\right]+\nu \frac{\partial}{\partial \mathbf{P}} \int_{n+1} \Phi_{b}^{\prime}\left(\mathbf{R}-\mathbf{r}_{n+1}\right) F_{n+1}+} \\
& +\nu \sum_{j=1}^{n} \frac{\partial}{\partial \mathbf{p}_{j}} \int_{n+1} \Phi_{a}^{\prime}\left(\mathbf{r}_{j}-\mathbf{r}_{n+1}\right) F_{n+1}, \quad n=0,1, \ldots,
\end{aligned}
$$

с начальными условиями

$$
\begin{aligned}
& \left.F_{0}(t)\right|_{t=0}=\delta\left(\mathbf{R}-\mathbf{R}_{0}\right) G_{M}(\mathbf{P}), \\
& \left.F_{n}(t)\right|_{t=0}=\delta\left(\mathbf{R}-\mathbf{R}_{0}\right) F_{n}^{(\mathrm{eq})}\left(\mathbf{r}^{(n)} \mid \mathbf{R} ; \nu\right) G_{M}(\mathbf{P}) \prod_{j=1}^{n} G_{m}\left(\mathbf{p}_{j}\right),
\end{aligned}
$$

где $H_{n}$ - гамильтониан подсистемы, состоящей из $n$ атомов и БЧ, $\Phi_{a, b}^{\prime}(\mathbf{r})=\nabla \Phi_{a, b}(\mathbf{r})$, а $F_{n}^{(\text {eq) }}\left(\mathbf{r}^{(n)} \mid \mathbf{R} ; \nu\right)$ - обычные термодинамически равновесные $\Phi$ Р $n$ атомов в конфигурационном пространстве в присутствии БЧ, находящейся в точке $\mathbf{R}$.

В принципе этого достаточно, чтобы без каких-либо дополнительных предположений найти функцию $F_{0}\left(t, \mathbf{R}, \mathbf{P} \mid \mathbf{R}_{0} ; \nu\right)$ и тем самым вероятностное распределение перемещения БЧ $\Delta \mathbf{R}=\mathbf{R}-\mathbf{R}_{0}$. Но, как известно, решение задач, связанных с рекуррентными соотношениями, может быть облегчено использованием производящих функций. Применительно к цепочке ББГКИ такой подход был сформулирован и опробован Боголюбовым [8]. Нам он поможет выявить такие свойства решений этой цепочки, которые трудно увидеть непосредственно из нее. Следуя Боголюбову, объединим все наши ФР в производящий функционал

$$
\mathcal{F}\left\{t, \mathbf{R}, \mathbf{P}, \psi \mid \mathbf{R}_{0} ; \nu\right\}=F_{0}+\sum_{n=1}^{\infty} \frac{\nu^{n}}{n !} \int_{1} \ldots \int_{n} F_{n} \prod_{j=1}^{n} \psi\left(\mathbf{r}_{j}, \mathbf{p}_{j}\right),
$$

а уравнения (2.2) - в соответствующее производящее уравнение для него

$$
\frac{\partial \mathcal{F}}{\partial t}+\frac{\mathbf{P}}{M} \cdot \frac{\partial \mathcal{F}}{\partial \mathbf{R}}=\widehat{\mathcal{L}}\left(\psi, \frac{\delta}{\delta \psi}\right) \mathcal{F}
$$

с оператором $\widehat{\mathcal{L}}$, составленным из обычных и вариационных производных,

$$
\begin{aligned}
\widehat{\mathcal{L}}\left(\psi, \frac{\delta}{\delta \psi}\right)= & -\int_{1} \psi\left(x_{1}\right) \frac{\mathbf{p}_{1}}{m} \cdot \frac{\partial}{\partial \mathbf{r}_{1}} \frac{\delta}{\delta \psi\left(x_{1}\right)}+\int_{1}\left[1+\psi\left(x_{1}\right)\right]\left[\Phi_{b}\left(\mathbf{R}-\mathbf{r}_{1}\right), \frac{\delta}{\delta \psi\left(x_{1}\right)}\right]+ \\
& +\frac{1}{2} \int_{1} \int_{2}\left[1+\psi\left(x_{1}\right)\right]\left[1+\psi\left(x_{2}\right)\right]\left[\Phi_{a}\left(\mathbf{r}_{1}-\mathbf{r}_{2}\right), \frac{\delta^{2}}{\delta \psi\left(x_{1}\right) \delta \psi\left(x_{2}\right)}\right]
\end{aligned}
$$

где $x_{j}=\left\{\mathbf{r}_{j}, \mathbf{p}_{j}\right\}$. Данное уравнение - прямой аналог уравнения (7.9) из монографии [8]. Чтобы продемонстрировать это, заметим, что $\psi(x)=u(x) / \nu$, где $u(x)$ - функциональный аргумент, использованный в монографии [8], и что фактор 
$\left[1+\psi\left(x_{1}\right)\right]\left[1+\psi\left(x_{2}\right)\right]$ в $(2.6)$ можно заменить на $\left[\psi\left(x_{1}\right)+\psi\left(x_{2}\right)+\psi\left(x_{1}\right) \psi\left(x_{2}\right)\right]$ благодаря тождеству $\int_{1} \int_{2}\left[\Phi_{a}\left(\mathbf{r}_{1}-\mathbf{r}_{2}\right), \ldots\right]=0$.

Запишем начальное условие к уравнению (2.5). Для этого введем производящий функционал равновесных $Ф$ в в конфигурационном пространстве

$$
\mathcal{F}^{(\mathrm{eq})}\{\phi \mid \mathbf{R} ; \nu\}=1+\sum_{n=1}^{\infty} \frac{\nu^{n}}{n !} \int \ldots \int F_{n}^{(\mathrm{eq})}\left(\mathbf{r}^{(n)} \mid \mathbf{R} ; \nu\right) \prod_{j=1}^{n} \phi\left(\mathbf{r}_{j}\right) d \mathbf{r}_{j},
$$

где $\phi(\mathbf{r})$ - соответствующая функциональная переменная. Кроме того, введем линейное отображение $\phi\{\psi\}$ функций $\psi(\mathbf{r}, \mathbf{p})$ в функции $\phi(\mathbf{r})$ :

$$
\phi\{\psi\}(\mathbf{r})=\int \psi(\mathbf{r}, \mathbf{p}) G_{m}(\mathbf{p}) d \mathbf{p} .
$$

Тогда начальные условия (2.3) объединяются в следующее условие:

$$
\mathcal{F}\left\{0, \mathbf{R}, \mathbf{P}, \psi \mid \mathbf{R}_{0} ; \nu\right\}=\delta\left(\mathbf{R}-\mathbf{R}_{0}\right) G_{M}(\mathbf{P}) \mathcal{F}^{(\mathrm{eq})}\{\phi\{\psi\} \mid \mathbf{R} ; \nu\} .
$$

Легко догадаться (или проверить), что комбинация $G_{M}(\mathbf{P}) \mathcal{F}^{(\mathrm{eq})}\{\phi\{\psi\} \mid \mathbf{R} ; \nu\}$ доставляет уравнению (2.5) стационарное решение, так что

$$
\left[-\frac{\mathbf{P}}{M} \cdot \frac{\partial}{\partial \mathbf{R}}+\widehat{\mathcal{L}}\right] G_{M}(\mathbf{P}) \mathcal{F}^{(\mathrm{eq})}\{\phi\{\psi\} \mid \mathbf{R} ; \nu\}=0 .
$$

Отсюда вытекает уравнение

$$
\left[\frac{\partial}{\partial \mathbf{r}}+\frac{\Phi_{b}^{\prime}(\mathbf{r}-\mathbf{R})}{T}\right] \frac{\delta \mathcal{F}^{(\mathrm{eq})}}{\delta \phi(\mathbf{r})}=\frac{1}{T} \int\left[1+\phi\left(\mathbf{r}^{\prime}\right)\right] \Phi_{a}^{\prime}\left(\mathbf{r}^{\prime}-\mathbf{r}\right) \frac{\delta^{2} \mathcal{F}^{(\mathrm{eq})}}{\delta \phi(\mathbf{r}) \delta \phi\left(\mathbf{r}^{\prime}\right)} d \mathbf{r}^{\prime},
$$

в котором, как и в $(2.7), \phi(\mathbf{r})$ фигурирует как самостоятельная функциональная переменная в конфигурационном пространстве. Это уравнение определяет равновесные ФР и является аналогом уравнения (2.14) из монографии [8].

K сожалению, нестационарные решения производящих уравнений вида (2.5) или (7.9) из монографии [8], насколько нам известно, не исследовались. Однако опыт работы с уравнениями ББГКИ подсказывает (см., например, [10]) желательность замены переменных - перехода от ФР к подходящим функциям, позволяющим сосредоточиться на статистических зависимостях и корреляциях между частицами. С этой целью обсудим гипотетические равенства

$$
F_{n}\left(t, \mathbf{R}, \mathbf{r}^{(n)}, \mathbf{P}, \mathbf{p}^{(n)} \mid \mathbf{R}_{0} ; \nu\right) \stackrel{?}{=} F_{0}\left(t, \mathbf{R}, \mathbf{P} \mid \mathbf{R}_{0} ; \nu\right) F_{n}^{(\mathrm{eq})}\left(\mathbf{r}^{(n)} \mid \mathbf{R} ; \nu\right) \prod_{j=1}^{n} G_{m}\left(\mathbf{p}_{j}\right)
$$

или, что то же самое,

$$
\mathcal{F}\left\{t, \mathbf{R}, \mathbf{P}, \psi \mid \mathbf{R}_{0} ; \nu\right\} \stackrel{?}{=} F_{0}\left(t, \mathbf{R}, \mathbf{P} \mid \mathbf{R}_{0} ; \nu\right) \mathcal{F}^{(\mathrm{eq})}\{\phi\{\psi\} \mid \mathbf{R} ; \nu\} .
$$

Здесь утверждается, очевидно, что условные $\Phi$ Р атомов, т.е. $F_{n} / F_{0}$, не зависят от перемещения БЧ $\mathbf{R}-\mathbf{R}_{0}$. Данное предположение выглядит естественным, поскольку речь идет о термодинамически равновесном блуждании, при котором любые позиции БЧ равноправны. Тем не менее это ошибочное предположение, поскольку оно несовместимо с уравнениями (2.2). Так, при подстановке выражения 
$F_{0}\left(t, \mathbf{R}, \mathbf{P} \mid \mathbf{R}_{0} ; \nu\right) F_{1}^{(\mathrm{eq})}\left(\mathbf{r}_{1} \mid \mathbf{R} ; \nu\right) G_{m}\left(\mathbf{p}_{1}\right)$ в роли $F_{1}$ в уравнение для $F_{0}$ интеграл взаимодействия БЧ с атомами обращается в нуль: $\int_{1} \Phi_{b}^{\prime}\left(\mathbf{R}-\mathbf{r}_{1}\right) F_{1}=0$ (ввиду хотя бы того, что $F_{1}^{(\text {eq })}\left(\mathbf{r}_{1} \mid \mathbf{R} ; \nu\right)$ есть четная функция разности $\mathbf{r}_{1}-\mathbf{R}$, тогда как $\Phi_{b}^{\prime}\left(\mathbf{R}-\mathbf{r}_{1}\right)-$ нечетная функция). В результате первое из уравнений (2.2) сводится к уравнению свободного движения БЧ, $\partial F_{0} / \partial t=\left[H_{0}, F_{0}\right]$, как если бы БЧ вообще не взаимодействовала с атомами.

Из сказанного следует, что, во-первых, текущее состояние среды (системы атомов) статистически зависит от суммарного перемещения БЧ, $\Delta \mathbf{R}(t)=\mathbf{R}(t)-\mathbf{R}_{0}$, за все время ее наблюдения. Иными словами, существуют корреляции между средой и историей блуждания БЧ. Мы будем называть их историческими корреляииями, чтобы мысленно отделять их от присутствующих в $F_{n}^{(\text {eq) }}\left(\mathbf{r}^{(n)} \mid \mathbf{R} ; \nu\right)$ равновесных корреляций между текущими координатами БЧ и атомов. Во-вторых, мы адекватным образом формально разделим те и другие, если в качестве характеристик исторических корреляций возьмем функции $V_{n}=V_{n}\left(t, \mathbf{R}, \mathbf{r}^{(n)}, \mathbf{P}, \mathbf{p}^{(n)} \mid \mathbf{R}_{0} ; \nu\right)$, которые определяются следующими производящими соотношениями:

$$
\begin{aligned}
& \mathcal{F}\left\{t, \mathbf{R}, \mathbf{P}, \psi \mid \mathbf{R}_{0} ; \nu\right\}=\mathcal{V}\left\{t, \mathbf{R}, \mathbf{P}, \psi \mid \mathbf{R}_{0} ; \nu\right\} \mathcal{F}^{(\mathrm{eq})}\{\phi\{\psi\} \mid \mathbf{R} ; \nu\}, \\
& \mathcal{V}\left\{t, \mathbf{R}, \mathbf{P}, \psi \mid \mathbf{R}_{0} ; \nu\right\}=V_{0}+\sum_{n=1}^{\infty} \frac{\nu^{n}}{n !} \int_{1} \ldots \int_{n} V_{n} \prod_{j=1}^{n} \psi\left(\mathbf{r}_{j}, \mathbf{p}_{j}\right) .
\end{aligned}
$$

В частности, $V_{0}\left(t, \mathbf{R}, \mathbf{P} \mid \mathbf{R}_{0} ; \nu\right)=F_{0}\left(t, \mathbf{R}, \mathbf{P} \mid \mathbf{R}_{0} ; \nu\right)$ и

$$
\begin{aligned}
& F_{1}\left(t, \mathbf{R}, \mathbf{r}_{1}, \mathbf{P}, \mathbf{p}_{1} \mid \mathbf{R}_{0} ; \nu\right)= \\
& \quad=F_{0}\left(t, \mathbf{R}, \mathbf{P} \mid \mathbf{R}_{0} ; \nu\right) F_{1}^{(\mathrm{eq})}\left(\mathbf{r}_{1} \mid \mathbf{R} ; \nu\right) G_{m}\left(\mathbf{p}_{1}\right)+V_{1}\left(t, \mathbf{R}, \mathbf{r}_{1}, \mathbf{P}, \mathbf{p}_{1} \mid \mathbf{R}_{0} ; \nu\right) .
\end{aligned}
$$

Из данного определения видно, что с точки зрения теории вероятностей $V_{n}$ (при $n>0)$ представляют собой род кумулянтов. Поэтому мы будем называть их кумулянтными функциями $(\mathrm{K} \Phi)^{1)}$.

В терминах КФ начальные условия (2.3) или (2.9) значительно упрощаются:

$$
\mathcal{V}\left\{0, \mathbf{R}, \mathbf{P}, \psi \mid \mathbf{R}_{0} ; \nu\right\}=\delta\left(\mathbf{R}-\mathbf{R}_{0}\right) G_{M}(\mathbf{P}),
$$

т.е.

$$
\begin{gathered}
V_{0}\left(0, \mathbf{R}, \mathbf{P} \mid \mathbf{R}_{0} ; \nu\right)=\delta\left(\mathbf{R}-\mathbf{R}_{0}\right) G_{M}(\mathbf{P}), \\
V_{n}\left(0, \mathbf{R}, \mathbf{r}^{(n)}, \mathbf{P}, \mathbf{p}^{(n)} \mid \mathbf{R}_{0} ; \nu\right)=0, \quad n>0 .
\end{gathered}
$$

Упрощаются и условия ослабления корреляций:

$$
V_{n}\left(t, \mathbf{R}, \mathbf{r}^{(n)}, \mathbf{P}, \mathbf{p}^{(n)} \mid \mathbf{R}_{0} ; \nu\right) \rightarrow 0 \quad \text { при } \quad \mathbf{r}_{k} \rightarrow \infty, \quad 1 \leqslant k \leqslant n,
$$

т.е. КФ обращаются в нуль, если хотя бы один из атомов отдаляется от БЧ на бесконечность. Напротив, производящее уравнение (2.5) в терминах КФ усложняется. Подставляя (2.11) в (2.5), получаем

$$
\frac{\partial \mathcal{V}}{\partial t}+\frac{\mathbf{P}}{M} \cdot \frac{\partial \mathcal{V}}{\partial \mathbf{R}}=\widehat{\mathcal{L}}\left(\psi, \frac{\delta}{\delta \psi}\right) \mathcal{V}+\widehat{\mathcal{L}}^{\prime}\left(\nu, \psi, \frac{\delta}{\delta \psi}\right) \mathcal{V}
$$

\footnotetext{
1)В физической литературе (см., например, [10]) подобные объекты по традиции обычно именуются корреляционными функциями.
} 

где

$$
\begin{aligned}
\widehat{\mathcal{L}^{\prime}}\left(\nu, \psi, \frac{\delta}{\delta \psi}\right)= & \left\{\int[1+\phi(\mathbf{r})] \Phi_{b}^{\prime}(\mathbf{R}-\mathbf{r}) \nu \mathcal{C}\{\mathbf{r}, \phi \mid \mathbf{R} ; \nu\} d \mathbf{r}\right\}\left(\frac{\mathbf{P}}{M T}+\frac{\partial}{\partial \mathbf{P}}\right)+ \\
& +\int_{1} \int_{2}\left[1+\psi\left(x_{1}\right)\right]\left[1+\psi\left(x_{2}\right)\right] \times \\
& \times\left[\Phi_{a}\left(\mathbf{r}_{1}-\mathbf{r}_{2}\right), \nu \mathcal{C}\left\{\mathbf{r}_{2}, \phi \mid \mathbf{R} ; \nu\right\} G_{m}\left(\mathbf{p}_{2}\right) \frac{\delta}{\delta \psi\left(x_{1}\right)}\right] .
\end{aligned}
$$

Здесь $\phi(\mathbf{r})$ и $\phi$ понимаются как линейные функционалы $\psi(\mathbf{r}, \mathbf{p})$ в смысле отображения $\phi\{\psi\}(\mathbf{r})$, определенного формулой (2.8), и введен еще один функционал

$$
\mathcal{C}\{\mathbf{r}, \phi \mid \mathbf{R} ; \nu\}=\frac{\delta \ln \mathcal{F}^{(\mathrm{eq})}\{\phi \mid \mathbf{R} ; \nu\}}{\nu \delta \phi(\mathbf{r})} .
$$

Соответственно усложняются уравнения ББГКИ. Мы выпишем их только для крайнего, но принципиально интересного случая БЧ в идеальном газе (атомы взаимодействуют с БЧ, но не взаимодействуют друг с другом, т.е. $\left.\Phi_{a}(\mathbf{r})=0\right)$ :

$$
\begin{aligned}
\frac{\partial V_{0}}{\partial t}= & -\frac{\mathbf{P}}{M} \cdot \frac{\partial V_{0}}{\partial \mathbf{R}}+\nu \frac{\partial}{\partial \mathbf{P}} \int_{1} \Phi_{b}^{\prime}\left(\mathbf{R}-\mathbf{r}_{1}\right) V_{1} \\
\frac{\partial V_{n}}{\partial t}= & {\left[H_{n}, V_{n}\right]+\nu \frac{\partial}{\partial \mathbf{P}} \int_{n+1} \Phi_{b}^{\prime}\left(\mathbf{R}-\mathbf{r}_{n+1}\right) V_{n+1}+} \\
& +T \sum_{j=1}^{n} \mathcal{P}(j, n) G_{m}\left(\mathbf{p}_{n}\right) E^{\prime}\left(\mathbf{r}_{n}-\mathbf{R}\right)\left(\frac{\mathbf{P}}{M T}+\frac{\partial}{\partial \mathbf{P}}\right) V_{n-1}, \quad n>0,
\end{aligned}
$$

где

$$
\begin{gathered}
H_{n}=\frac{\mathbf{P}^{2}}{2 M}+\sum_{j=1}^{n}\left[\frac{\mathbf{p}_{j}^{2}}{2 m}+\Phi_{b}\left(\mathbf{R}-\mathbf{r}_{j}\right)\right] \\
E(\mathbf{r})=e^{-\Phi(\mathbf{r}) / T}, \quad E^{\prime}(\mathbf{r})=\nabla E(\mathbf{r})=-\frac{\Phi^{\prime}(\mathbf{r}) E(\mathbf{r})}{T},
\end{gathered}
$$

а $\mathcal{P}(j, n)$ обозначает операцию перестановки аргументов $x_{j}=\left\{\mathbf{r}_{j}, \mathbf{p}_{j}\right\}$ и $x_{n}=\left\{\mathbf{r}_{n}, \mathbf{p}_{n}\right\}$. Очевидно, в представлении через КФ цепочка ББГКИ из двухдиагональной превращается как минимум в трехдиагональную.

\section{3. ГРУППА ИНВАРИАНТНОСТИ РАВНОВЕСНОГО ПРОИЗВОДЯЩЕГО ФУНКЦИОНАЛА}

Рассмотрим функционал (2.16), который интересен уже потому, что входит в уравнение эволюции (2.15). Разлагая его в ряд с учетом (2.7), имеем

$$
\begin{aligned}
\mathcal{C}\{\mathbf{r}, \phi \mid \mathbf{R} ; \nu\}= & F_{1}^{(\mathrm{eq})}(\mathbf{r} \mid \mathbf{R} ; \nu)+ \\
& +\sum_{n=1}^{\infty} \frac{\nu^{n}}{n !} \int \ldots \int C_{n+1}\left(\mathbf{r}, \mathbf{r}_{1}, \ldots, \mathbf{r}_{n} \mid \mathbf{R} ; \nu\right) \prod_{j=1}^{n} \phi\left(\mathbf{r}_{j}\right) d \mathbf{r}_{j} .
\end{aligned}
$$


Тем самым определены функции $C_{n}\left(\mathbf{r}_{1}, \ldots, \mathbf{r}_{n} \mid \mathbf{R} ; \nu\right), n=2,3, \ldots$. В частности,

$$
\begin{aligned}
C_{2}\left(\mathbf{r}, \mathbf{r}_{1} \mid \mathbf{R} ; \nu\right)= & F_{2}^{(\mathrm{eq})}\left(\mathbf{r}, \mathbf{r}_{1} \mid \mathbf{R} ; \nu\right)-F_{1}^{(\mathrm{eq})}(\mathbf{r} \mid \mathbf{R} ; \nu) F_{1}^{(\mathrm{eq})}\left(\mathbf{r}_{1} \mid \mathbf{R} ; \nu\right) \\
C_{3}\left(\mathbf{r}, \mathbf{r}_{1}, \mathbf{r}_{2}\right)= & F_{3}^{(\mathrm{eq})}\left(\mathbf{r}, \mathbf{r}_{1}, \mathbf{r}_{2}\right)+2 F_{1}^{(\mathrm{eq})}(\mathbf{r}) F_{1}^{(\mathrm{eq})}\left(\mathbf{r}_{1}\right) F_{1}^{(\mathrm{eq})}\left(\mathbf{r}_{2}\right)- \\
& -F_{1}^{(\mathrm{eq})}(\mathbf{r}) F_{2}^{(\mathrm{eq})}\left(\mathbf{r}_{1}, \mathbf{r}_{2}\right)-F_{1}^{(\mathrm{eq})}\left(\mathbf{r}_{1}\right) F_{2}^{(\mathrm{eq})}\left(\mathbf{r}, \mathbf{r}_{2}\right)-F_{1}^{(\mathrm{eq})}\left(\mathbf{r}_{2}\right) F_{2}^{(\mathrm{eq})}\left(\mathbf{r}, \mathbf{r}_{1}\right)
\end{aligned}
$$

(во втором выражении для краткости аргументы $\mathbf{R}$ и $\nu$ опущены). Отсюда ясно, что с точки зрения атомных координат функции $C_{n}$ относятся к равновесным $\Phi \mathrm{P} F_{n}^{(\mathrm{eq})}$ точно так, как кумулянты случайного поля относятся к его статистическим моментам. Кластерное свойство означает, что все они исчезают при удалении хотя бы одного из атомов от других:

$$
C_{n}\left(\mathbf{r}_{1}, \ldots, \mathbf{r}_{n} \mid \mathbf{R} ; \nu\right) \rightarrow 0 \quad \text { при } \quad \mathbf{r}_{j} \rightarrow \infty .
$$

При удалении же БЧ от атомов имеем

$$
\begin{aligned}
F_{1}^{(\text {eq })}(\mathbf{r} \mid \mathbf{R} ; \nu) & \rightarrow 1 & \text { при } & \mathbf{R} \rightarrow \infty, \\
C_{n}\left(\mathbf{r}_{1}, \ldots, \mathbf{r}_{n} \mid \mathbf{R} ; \nu\right) & \rightarrow C_{n}\left(\mathbf{r}_{1}, \ldots, \mathbf{r}_{n} ; \nu\right) & \text { при } & \mathbf{R} \rightarrow \infty
\end{aligned}
$$

где $C_{n}\left(\mathbf{r}_{1}, \ldots, \mathbf{r}_{n} ; \nu\right)$ - кумулянты равновесной среды в отсутствие БЧ. Более того, при достаточно короткодействующих потенциалах все это происходит быстро, абсолютно интегрируемым образом, что далее предполагается.

Обратимся теперь к уравнению (2.10), переписав его в форме

$$
\begin{aligned}
& {\left[\frac{\partial}{\partial \mathbf{r}}+\frac{\Phi_{b}^{\prime}(\mathbf{r}-\mathbf{R})}{T}\right] \mathcal{C}\{\mathbf{r}, \phi \mid \mathbf{R} ; \nu\}=\frac{1}{T} \int\left[1+\phi\left(\mathbf{r}^{\prime}\right)\right] \Phi_{a}^{\prime}\left(\mathbf{r}^{\prime}-\mathbf{r}\right) \frac{\delta \mathcal{C}\left\{\mathbf{r}^{\prime}, \phi \mid \mathbf{R} ; \nu\right\}}{\delta \phi(\mathbf{r})} d \mathbf{r}^{\prime}+} \\
& \quad+\mathcal{C}\{\mathbf{r}, \phi \mid \mathbf{R} ; \nu\} \frac{\nu}{T} \int\left[1+\phi\left(\mathbf{r}^{\prime}\right)\right] \Phi_{a}^{\prime}\left(\mathbf{r}^{\prime}-\mathbf{r}\right) \mathcal{C}\left\{\mathbf{r}^{\prime}, \phi \mid \mathbf{R} ; \nu\right\} d \mathbf{r}^{\prime}
\end{aligned}
$$

и с его помощью выявим (вероятно, впервые) некоторые важные свойства функционала $\mathcal{C}\{\mathbf{r}, \phi \mid \mathbf{R} ; \nu\}$. Во-первых, заметим, что благодаря интегрируемости асимптотик (3.2) этот функционал можно распространить на ограниченные функции $\phi(\mathbf{r})$, которые не обращаются в нуль на бесконечности, в частности на константы. Данное обстоятельство позволяет ввести следующие объекты:

$$
\begin{gathered}
C(\sigma, \nu)=\lim _{\mathbf{R} \rightarrow \infty} \mathcal{C}\{\mathbf{r}, \sigma \mid \mathbf{R} ; \nu\}=1+\sum_{n=1}^{\infty} \frac{\nu^{n} \sigma^{n}}{n !} \int_{1} \ldots \int_{n} C_{n+1}\left(\mathbf{r}, \mathbf{r}_{1}, \ldots, \mathbf{r}_{n} ; \nu\right), \\
\mathcal{C}_{\sigma}\{\mathbf{r}, \phi \mid \mathbf{R} ; \nu\}=\frac{\mathcal{C}\{\mathbf{r}, \sigma+\phi \mid \mathbf{R} ; \nu\}}{C(\sigma, \nu)}
\end{gathered}
$$

где $\sigma=$ const, $\int_{n} \cdots=\int \ldots d \mathbf{r}_{n}$, а интегралы в (3.5) фактически не зависят от $\mathbf{r}$. Во-вторых, после замены $\phi(\mathbf{r}) \rightarrow \sigma+\phi(\mathbf{r})$ уравнение (3.4) элементарными алгебраическими манипуляциями преобразуется в уравнение для $\mathcal{C}_{\sigma}\{\mathbf{r}, \phi \mid \mathbf{R} ; \nu\}$ :

$$
\begin{aligned}
{\left[\frac{\partial}{\partial \mathbf{r}}\right.} & \left.+\frac{\Phi_{b}^{\prime}(\mathbf{r}-\mathbf{R})}{T}\right] \mathcal{C}_{\sigma}\{\mathbf{r}, \phi \mid \mathbf{R} ; \nu\}= \\
& =\frac{1}{T} \int\left[1+\frac{\phi\left(\mathbf{r}^{\prime}\right)}{1+\sigma}\right] \Phi_{a}^{\prime}\left(\mathbf{r}^{\prime}-\mathbf{r}\right) \frac{\delta \mathcal{C}_{\sigma}\left\{\mathbf{r}^{\prime}, \phi \mid \mathbf{R} ; \nu\right\}}{\delta[\phi(\mathbf{r}) /(1+\sigma)]} d \mathbf{r}^{\prime}+
\end{aligned}
$$




$$
\begin{aligned}
& +\mathcal{C}_{\sigma}\{\mathbf{r}, \phi \mid \mathbf{R} ; \nu\} \frac{\nu C(\sigma, \nu)(1+\sigma)}{T} \times \\
& \times \int\left[1+\frac{\phi\left(\mathbf{r}^{\prime}\right)}{1+\sigma}\right] \Phi_{a}^{\prime}\left(\mathbf{r}^{\prime}-\mathbf{r}\right) \mathcal{C}_{\sigma}\left\{\mathbf{r}^{\prime}, \phi \mid \mathbf{R} ; \nu\right\} d \mathbf{r}^{\prime}
\end{aligned}
$$

Легко увидеть, что оно отличается от (3.4) только масштабным преобразованием функционального аргумента, $\phi(\mathbf{r}) \rightarrow \phi(\mathbf{r}) /(1+\sigma)$, и заменой плотности $\nu$ на

$$
v(\sigma, \nu)=\nu C(\sigma, \nu)(1+\sigma) .
$$

В-третьих, формальное решение уравнения (3.4) в виде ряда (3.1) однозначно определяется условиями ослабления корреляций (3.2) и (3.3). В-четвертых, по определению (3.5), (3.6) функционала $\mathcal{C}_{\sigma}\{\mathbf{r}, \phi \mid \mathbf{R} ; \nu\}$ коэффициенты его разложения в ряд по $\phi(\mathbf{r})$ удовлетворяют таким же условиям. А именно, из $(3.2),(3.3)$ следует, что

$$
\begin{aligned}
& \frac{1}{C(\sigma, \nu)}\left[F_{1}^{(\text {eq })}(\mathbf{r} \mid \mathbf{R} ; \nu)+\right. \\
& \left.\quad+\sum_{k=1}^{\infty} \frac{\nu^{k} \sigma^{k}}{k !} \int_{1} \ldots \int_{k} C_{k+1}\left(\mathbf{r}, \mathbf{r}_{1}, \ldots, \mathbf{r}_{k} \mid \mathbf{R} ; \nu\right)\right] \rightarrow 1 \quad \text { при } \quad \mathbf{R} \rightarrow \infty \\
& C_{n}\left(\mathbf{r}_{1}, \ldots, \mathbf{r}_{n} \mid \mathbf{R} ; \nu\right)+ \\
& \quad+\sum_{k=1}^{\infty} \frac{\nu^{k} \sigma^{k}}{k !} \int_{n+1} \ldots \int_{n+k} C_{n+k}\left(\mathbf{r}_{1}, \ldots, \mathbf{r}_{n+k} \mid \mathbf{R} ; \nu\right) \rightarrow 0 \quad \text { при } \quad \mathbf{r}_{j} \rightarrow \infty
\end{aligned}
$$

где $1 \leqslant j \leqslant n$, по крайней мере если понимать условия (3.2), (3.3) в смысле абсолютной интегрируемости и говорить о не слишком больших значениях плотности (подробнее см. [9]).

Суммируя вышесказанное, приходим к выводу, что решение уравнения (3.7) это не что иное, как $\mathcal{C}_{\sigma}\{\mathbf{r}, \phi \mid \mathbf{R}, \nu\}=\mathcal{C}\{\mathbf{r}, \phi /(1+\sigma) \mid \mathbf{R} ; v(\sigma, \nu)\}$. Объединяя данный результат с (3.6) и (3.8), получаем, что для произвольных (в определенном смысле) константы $\sigma$ и ограниченной функции $\phi=\phi(\mathbf{r})$ имеет место равенство

$$
\nu \mathcal{C}\{\mathbf{r}, \sigma+\phi \mid \mathbf{R} ; \nu\}=\frac{v(\sigma, \nu)}{1+\sigma} \mathcal{C}\left\{\mathbf{r}, \frac{\phi}{1+\sigma} \mid \mathbf{R} ; v(\sigma, \nu)\right\} .
$$

Его можно переписать еще в виде

$$
\widehat{\mathcal{T}}(\sigma) \mathcal{C}\{\mathbf{r}, \phi \mid \mathbf{R} ; \nu\} \equiv \frac{v(\sigma, \nu)}{(1+\sigma) \nu} \mathcal{C}\left\{\mathbf{r}, \frac{1+\phi}{1+\sigma}-1 \mid \mathbf{R} ; v(\sigma, \nu)\right\}=\mathcal{C}\{\mathbf{r}, \phi \mid \mathbf{R} ; \nu\} .
$$

Здесь первое равенство служит определением однопараметрического семейства таких преобразований функционала $\mathcal{C}\{\mathbf{r}, \phi \mid \mathbf{R} ; \nu\}$, которые согласно второму равенству сохраняют его значение. Несложно проверить, что это группа с правилом умножения

$$
\begin{aligned}
\widehat{\mathcal{T}}\left(\sigma_{2}\right) \widehat{\mathcal{T}}\left(\sigma_{1}\right) & =\widehat{\mathcal{T}}\left(\sigma_{1}+\sigma_{2}+\sigma_{1} \sigma_{2}\right), \\
v\left(\sigma_{2}, v\left(\sigma_{1}, \nu\right)\right) & =v\left(\sigma_{1}+\sigma_{2}+\sigma_{1} \sigma_{2}, \nu\right)
\end{aligned}
$$

и с ограничениями $\sigma>-1, \phi(\mathbf{r})>-1$. Упомянутые ограничения понятны в свете того, что функционал $\nu[1+\phi(\mathbf{r})] \mathcal{C}\{\mathbf{r}, \phi \mid \mathbf{R} ; \nu\}$ представляет собой среднюю концентрацию атомов в присутствии стороннего потенциала $U(\mathbf{r})$, связанного с $\phi(\mathbf{r})$ 
соотношением $\phi(\mathbf{r})=e^{-U(\mathbf{r}) / T}-1$ (см., например, [11]). Замена $\sigma=e^{a}-1$ дает $\widehat{\mathcal{T}}\left(a_{2}\right) \widehat{\mathcal{T}}\left(a_{1}\right)=\widehat{\mathcal{T}}\left(a_{1}+a_{2}\right)$, снимая ограничения.

Инфинитезимальную форму (3.9), (3.10) удобно представить через КФ:

$$
\begin{gathered}
\left\{\varkappa(\nu)+[1+\varkappa(\nu)] \nu \frac{\partial}{\partial \nu}\right\} F_{1}^{(\mathrm{eq})}(\mathbf{r} \mid \mathbf{R} ; \nu)=\nu \int C_{2}\left(\mathbf{r}, \mathbf{r}^{\prime} \mid \mathbf{R} ; \nu\right) d \mathbf{r}^{\prime}, \\
\left\{n \varkappa(\nu)+[1+\varkappa(\nu)] \nu \frac{\partial}{\partial \nu}\right\} C_{n}\left(\mathbf{r}_{1}, \ldots, \mathbf{r}_{n} \mid \mathbf{R} ; \nu\right)=\nu \int C_{n+1}\left(\mathbf{r}_{1}, \ldots, \mathbf{r}_{n}, \mathbf{r}^{\prime} \mid \mathbf{R} ; \nu\right) d \mathbf{r}^{\prime}, \\
\varkappa(\nu) \equiv\left[\frac{\partial C(\sigma, \nu)}{\partial \sigma}\right]_{\sigma=0}=\nu \int C_{2}(\mathbf{r}, 0 ; \nu) d \mathbf{r} .
\end{gathered}
$$

Функция $\varkappa(\nu)$, как известно (см., например, [12]), непосредственно связана с уравнением состояния системы: $1+\varkappa(\nu)=T(\partial \nu / \partial \mathcal{P})_{T}$, где $\mathcal{P}$ обозначает давление. Заметим, что в рамках большого канонического ансамбля близкие по содержанию соотношения легко получаются дифференцированием ФР по активности.

\section{4. ГРУППА ИНВАРИАНТНОСТИ ПРОИЗВОДЯЩЕГО ФУНКЦИОНАЛА ИСТОРИЧЕСКИХ КОРРЕЛЯЦИЙ}

Мы подошли к обоснованию основного результата настоящей работы. Покажем, что решение уравнения эволюции (2.15) обладает аналогичным (3.10) свойством инвариантности. Поскольку начальное условие к этому уравнению (см. (2.13)) вообще не зависит от переменных $\psi=\psi(\mathbf{r}, \mathbf{p})$ и $\nu$, решения уравнения $(2.15)$ целиком определяются структурой операторов $\widehat{\mathcal{L}}$ и $\widehat{\mathcal{L}}^{\prime}$ и условиями $(2.14)$ на бесконечности. Благодаря последним функционал $\mathcal{V}\left\{t, \mathbf{R}, \mathbf{P}, \psi \mid \mathbf{R}_{0} ; \nu\right\}$ (подобно функционалу $\mathcal{C}$ выше) можно распространить на аргумент $\sigma+\psi(\mathbf{r}, \mathbf{p})$, где $\sigma=$ const, вместо $\psi(\mathbf{r}, \mathbf{p})$. То, что скорость стремления к пределу в (2.14) достаточна для этого, будет ясно задним числом (см. ниже (4.7)). Далее, в силу (2.14) и определения (2.6) оператора $\widehat{\mathcal{L}}$ переменная $\psi\left(x_{1}\right)$ в первом члене выражения $\widehat{\mathcal{L}} \mathcal{V}$ может быть сдвинута на произвольную константу:

$$
\int_{1} \psi\left(x_{1}\right) \frac{\mathbf{p}_{1}}{m} \cdot \frac{\partial}{\partial \mathbf{r}_{1}} \frac{\delta \mathcal{V}}{\delta \psi\left(x_{1}\right)}=\int_{1}\left[a+\psi\left(x_{1}\right)\right] \frac{\mathbf{p}_{1}}{m} \cdot \frac{\partial}{\partial \mathbf{r}_{1}} \frac{\delta \mathcal{V}}{\delta \psi\left(x_{1}\right)}
$$

где $a=$ const, например $a=1$. В этом важное отличие $\widehat{\mathcal{L}} \mathcal{V}$ от $\widehat{\mathcal{L}} \mathcal{F}$. Как следствие, имея в виду действие $\widehat{\mathcal{L}}$ на $\mathcal{V}$, можно написать

$$
\widehat{\mathcal{L}}\left(\sigma+\psi, \frac{\delta}{\delta \psi}\right)=\widehat{\mathcal{L}}\left(\frac{\psi}{1+\sigma}, \frac{\delta}{\delta[\psi /(1+\sigma)]}\right) .
$$

Наконец, рассмотрим внимательно оператор $\widehat{\mathcal{L}}^{\prime}$ (см. (2.15)). В отличие от $\widehat{\mathcal{L}}$, он зависит от плотности $\nu$. Однако с учетом равенства (3.9) легко убедиться, что он подчиняется аналогичному (4.1) соотношению, если преобразование аргумента $\psi(\mathbf{r}, \mathbf{p})$ сопровождается преобразованием аргумента $\nu$ в соответствии с (3.8):

$$
\widehat{\mathcal{L}}^{\prime}\left(\nu, \sigma+\psi, \frac{\delta}{\delta \psi}\right)=\widehat{\mathcal{L}}^{\prime}\left(v(\sigma, \nu), \frac{\psi}{1+\sigma}, \frac{\delta}{\delta[\psi /(1+\sigma)]}\right) .
$$


Из приведенных выше рассуждений вытекает свойство инвариантности

$$
\mathcal{V}\left\{t, \mathbf{R}, \mathbf{P}, \sigma+\psi \mid \mathbf{R}_{0} ; \nu\right\}=\mathcal{V}\left\{t, \mathbf{R}, \mathbf{P}, \frac{\psi}{1+\sigma} \mid \mathbf{R}_{0} ; v(\sigma, \nu)\right\},
$$

или, эквивалентно и аналогично (3.10),

$$
\begin{aligned}
\widehat{\mathcal{T}}(\sigma) \mathcal{V}\left\{t, \mathbf{R}, \mathbf{P}, \psi \mid \mathbf{R}_{0} ; \nu\right\} & \equiv \mathcal{V}\left\{t, \mathbf{R}, \mathbf{P}, \frac{1+\psi}{1+\sigma}-1 \mid \mathbf{R}_{0} ; v(\sigma, \nu)\right\}= \\
& =\mathcal{V}\left\{t, \mathbf{R}, \mathbf{P}, \psi \mid \mathbf{R}_{0} ; \nu\right\},
\end{aligned}
$$

где первое равенство совместно с (3.5), (3.8), (3.10) и (3.11) определяет действие описанной выше группы преобразований на функционал $\mathcal{V}\left\{t, \mathbf{R}, \mathbf{P}, \psi \mid \mathbf{R}_{0} ; \nu\right\}$. Как видно, по отношению к нему это преобразования аргументов функционала, не меняющие его значения. Разложение (4.3) по степеням $\psi$ дает

$$
\begin{aligned}
& V_{0}\left(t, \mathbf{R}, \mathbf{P} \mid \mathbf{R}_{0} ; v(\sigma, \nu)\right)=V_{0}\left(t, \mathbf{R}, \mathbf{P} \mid \mathbf{R}_{0} ; \nu\right)+ \\
& \quad+\sum_{n=1}^{\infty} \frac{\nu^{n} \sigma^{n}}{n !} \int_{1} \ldots \int_{n} V_{n}\left(t, \mathbf{R}, \mathbf{r}^{(n)}, \mathbf{P}, \mathbf{p}^{(n)} \mid \mathbf{R}_{0} ; \nu\right), \\
& {\left[\frac{v(\sigma, \nu)}{(1+\sigma) \nu}\right]^{n} V_{n}\left(t, \mathbf{R}, \mathbf{r}^{(n)}, \mathbf{P}, \mathbf{p}^{(n)} \mid \mathbf{R}_{0} ; v(\sigma, \nu)\right)=V_{n}\left(t, \mathbf{R}, \mathbf{r}^{(n)}, \mathbf{P}, \mathbf{p}^{(n)} \mid \mathbf{R}_{0} ; \nu\right)+} \\
& \quad+\sum_{k=1}^{\infty} \frac{\nu^{k} \sigma^{k}}{k !} \int_{n+1} \ldots \int_{n+k} V_{n+k}\left(t, \mathbf{R}, \mathbf{r}^{(n+k)}, \mathbf{P}, \mathbf{p}^{(n+k)} \mid \mathbf{R}_{0} ; \nu\right) .
\end{aligned}
$$

Соответствующие инфинитезимальные по $\sigma$ соотношения подобны (3.12):

$$
\begin{gathered}
\left\{n \varkappa(\nu)+[1+\varkappa(\nu)] \nu \frac{\partial}{\partial \nu}\right\} V_{n}\left(t, \mathbf{R}, \mathbf{r}^{(n)}, \mathbf{P}, \mathbf{p}^{(n)} \mid \mathbf{R}_{0} ; \nu\right)= \\
=\nu \int_{n+1} V_{n+1}\left(t, \mathbf{R}, \mathbf{r}^{(n+1)}, \mathbf{P}, \mathbf{p}^{(n+1)} \mid \mathbf{R}_{0} ; \nu\right) .
\end{gathered}
$$

Формулы (4.5) и (4.6) могут трактоваться как вириальные разложения вероятностного распределения перемещения БЧ и статистических корреляций между перемещением БЧ и средой, с тем отличием от обычных вириальных разложений термодинамических величин [12] или кинетических коэффициентов [13], что вместо абсолютных значений плотности здесь фигурируют ее приращения. Впрочем, в пределе $\nu \rightarrow 0, \sigma \rightarrow \infty, \nu \sigma=$ const наши соотношения принимают более привычный вид. Например, (4.5) переходит в

$$
\begin{aligned}
& V_{0}\left(t, \mathbf{R}, \mathbf{P} \mid \mathbf{R}_{0} ; v(\nu)\right)= V_{0}\left(t, \mathbf{R}, \mathbf{P} \mid \mathbf{R}_{0} ; 0\right)+ \\
&+\sum_{n=1}^{\infty} \frac{\nu^{n}}{n !} \int V_{n}\left(t, \mathbf{R}, \mathbf{r}^{(n)}, \mathbf{P}, \mathbf{p}^{(n)} \mid \mathbf{R}_{0} ; 0\right), \\
& v(\nu) \equiv \nu\left[1+\sum_{n=1}^{\infty} \frac{\nu^{n}}{n !} \int_{1} \ldots \int_{n} C_{n+1}\left(\mathbf{r}, \mathbf{r}_{1}, \ldots, \mathbf{r}_{n} ; 0\right)\right],
\end{aligned}
$$


где интеграл в первом равенстве есть интеграл по всем атомным переменным. Следует заметить, что формула (4.5) ранее была получена в работах [11], [14], [15] исходя из "обобщенных флуктуационно-диссипационных соотношений" [16], [17]. До этого же, насколько нам известно, подобных точных соотношений в статистической физике не существовало. Весьма сложно было бы извлечь их непосредственно из уравнений ББГКИ (2.2) или эквивалентных уравнений для КФ. Исключением является специальный случай БЧ в идеальном газе, когда $C_{n}=0$ и соотношения (4.7) $($ с $\varkappa(\nu)=0)$ легко получаются с помощью дифференцирования уравнений (2.17) по плотности.

\section{5. О ПРИНЦИПИАЛЬНЫХ СЛЕДСТВИЯХ СООТНОШЕНИЙ ИНВАРИАНТНОСТИ}

Точное решение уравнений ББГКИ автоматически удовлетворяет всем вириальным разложениям (4.5)-(4.8), вытекающим из рассмотренной группы инвариантности. Более того, разложение (4.8) само представляет явное решение уравнений ББГКИ в виде формального ряда (так как при $\nu=0$ решение уравнений для КФ сводится к последовательным квадратурам). Поэтому естественно привлечь полученные результаты для проверки статистических гипотез о блуждании БЧ.

В этом смысле важно подчеркнуть, что формулы (4.5), (4.6) связывают между собой два случайных блуждания в средах с произвольно задаваемым отношением плотностей $v(\sigma, \nu) / \nu$. Следовательно, все члены ряда в (4.5) или (4.6) одинаково нужны (как и в (4.8)), и обрывание ряда дало бы не приближенный, а неправильный результат, даже в пределе разреженного газа (когда газовые параметры стремятся к нулю, $4 \pi r_{a}^{3} \nu / 3 \rightarrow 0,4 \pi r_{b}^{3} \nu / 3 \rightarrow 0$, где $r_{a, b}$ - радиусы двух взаимодействий) или в так называемом пределе Больцмана-Грэда (когда длины свободного пробега $\Lambda_{b}=\left(\pi r_{b}^{2} \nu\right)^{-1}$ и $\Lambda_{a}=\left(\pi r_{a}^{2} \nu\right)^{-1}$ фиксированы), как и в случае идеального газа (где $\Lambda_{a}=\infty$, но $\Lambda_{b}$ конечна). В этих случаях формулы (4.5)-(4.7) упрощаются и принимают вид

$$
\begin{aligned}
& \frac{\partial^{k} V_{n}\left(t, \mathbf{R}, \mathbf{r}^{(n)}, \mathbf{P}, \mathbf{p}^{(n)} \mid \mathbf{R}_{0} ; \nu\right)}{\partial \nu^{k}}= \\
& \quad=\int_{n+1} \cdots \int_{n+k} V_{n+k}\left(t, \mathbf{R}, \mathbf{r}^{(n+k)}, \mathbf{P}, \mathbf{p}^{(n+k)} \mid \mathbf{R}_{0} ; \nu\right),
\end{aligned}
$$

что делает совсем очевидным вывод, что с точки зрения точной теории все исторические корреляции всегда одинаково существенны.

Между тем в физической кинетике при выводе из уравнений ББГКИ замкнутых уравнений для одночастичных ФР (кинетических уравнений) принято отбрасывать межчастичные корреляции третьего и высших порядков (см., например, [8], [10], [13], [18]). Иногда, как в случае газа твердых сфер [18], [19], их незаметно теряют (см. [20]). Считается, что в пределе Больцмана-Грэда теория ББГКИ обязана сводиться к классической кинетической теории газов (где вероятностное распределение БЧ $F_{0}=V_{0}$ подчинялось бы линеаризованному уравнению Больцмана или аналогичному уравнению Больцмана-Лоренца [18], [19], [21]). Однако из вышесказанного следует, что на самом деле это не так: классическая кинетика не является предельным случаем теории, основанной на статистической механике. 
Для иллюстрации обратимся к БЧ в идеальном газе. Отбрасывая все высшие корреляции, т.е. полагая $V_{2}=0$ во втором $(n=1)$ из уравнений $(2.17)$ и подставляя его квадратуру в первое, придем к замкнутому уравнению для $V_{0}$. Асимптотика решения этого уравнения при $t \gg \tau=\Lambda_{b} / v_{0}$, где $v_{0} \sim \sqrt{T / M}-$ характерная тепловая скорость БЧ, заведомо гауссова:

$$
V_{0}(t, \Delta \mathbf{R} ; \nu) \equiv \int V_{0}\left(t, \mathbf{R}, \mathbf{P} \mid \mathbf{R}_{0} ; \nu\right) d \mathbf{P} \rightarrow \frac{e^{-\Delta \mathbf{R}^{2} / 4 D t}}{(4 \pi D t)^{3 / 2}},
$$

где $\Delta \mathbf{R}=\mathbf{R}-\mathbf{R}_{0}$, а $D=v_{0} \Lambda_{b}-$ коэффициент диффузии БЧ. Для разреженного газа $\Lambda_{b}=\left(\pi r_{b}^{2} \nu\right)^{-1}$ и $D \propto \nu^{-1}$. В любом случае (5.2) - весьма сложная функция $\nu$. В то же время с учетом точных соотношений (5.1) из $V_{2}=0$ следует, что $V_{0}(t, \Delta \mathbf{R} ; \nu)-$ чисто линейная функция $\nu$ !

Столь резкое несоответствие означает, что асимптотика (5.2) некорректна, и мы должны вернуться к полной цепочке ББГКИ. Приближенный подход к ее решению был предложен еще в работе [7] (см. также [21]) и развит в [22], где для случая, когда в роли БЧ выступает атом газа, была получена асимптотика

$$
V_{0}(t, \Delta \mathbf{R} ; \nu) \rightarrow \frac{\Gamma(7 / 2)}{[4 \pi D t]^{3 / 2}}\left[1+\frac{\Delta \mathbf{R}^{2}}{4 D t}\right]^{-7 / 2} \Theta\left(\frac{|\Delta \mathbf{R}|}{v_{0} t}\right),
$$

где $\Theta(x)$ - определенная функция, которая равна единице при $x=0$ и быстро стремится к нулю при $x \rightarrow \infty$. Характерной чертой данного распределения является то, что его кумулянты четвертого порядка растут со временем не линейно (как было бы в случае гауссовой асимптотики), а по закону $\propto(D t)^{2} \ln (t / \tau)$, т.е. приблизительно пропорционально квадрату времени. Эмпирически такое статистическое свойство случайного блуждания воспринимается как флуктуации текущего коэффициента диффузии БЧ [6], [7], [23], спектральная плотность которых на частотах $f \ll 1 / \tau$ растет приблизительно обратно пропорционально частоте (так называемый $1 / f$-шум [6]; таким образом, результаты [7], [21] подтвердили впервые высказанные в работах [23] догадки о происхождении $1 / f$-шума). С физической точки зрения причиной этих флуктуаций и соответствующих исторических статистических корреляций, описываемых $\mathrm{K} \Phi V_{n}(n>0)$, служит безразличие системы к истории столкновений БЧ: их количеству, частости (термин из [2]) и пропорциям чисел столкновений с разной величиной прицельного параметра [7]. Поэтому исторические корреляции обусловлены не столько взаимодействием частиц как таковым, сколько их соучастием во флуктуациях частости столкновений.

Покажем, что к аналогичным выводам можно прийти, опираясь лишь на вириальные разложения и на тот факт, что все $\Phi$ Р $F_{n}$ всегда неотрицательны. Для простоты ограничимся разреженным газом (газом Больцмана-Грэда). Из неотрицательности $F_{1}$ и тождества $(2.12)$ имеем

$$
V_{0}(t, \Delta \mathbf{R} ; \nu) \int_{\Omega} F_{1}^{(\mathrm{eq})}(\mathbf{r} \mid \mathbf{R} ; \nu) d \mathbf{r}+\int_{\Omega} V_{1}\left(t, \mathbf{R}, \mathbf{r} \mid \mathbf{R}_{0} ; \nu\right) d \mathbf{r} \geqslant 0
$$

где

$$
V_{1}\left(t, \mathbf{R}, \mathbf{r} \mid \mathbf{R}_{0} ; \nu\right) \equiv \iint V_{1}\left(t, \mathbf{R}, \mathbf{r}, \mathbf{P}, \mathbf{p} \mid \mathbf{R}_{0} ; \nu\right) d \mathbf{p} d \mathbf{P},
$$


а $\Omega$ - произвольная область в пространстве $\mathbf{r}-\mathbf{R}$. Введем $\Omega(\delta, t, \Delta \mathbf{R} ; \nu)=\Omega(\delta)$ как минимальную по объему из всех областей $\Omega$, удовлетворяющих условию

$$
\left|\int_{\Omega} V_{1} d \mathbf{r}-\int V_{1} d \mathbf{r}\right| \leqslant \delta\left|\int V_{1} d \mathbf{r}\right|,
$$

где $0<\delta<1$. Из этого определения следует, что

$$
\Omega \max _{\mathbf{r}}\left|V_{1}\right| \geqslant\left|\int_{\Omega} V_{1} d \mathbf{r}\right| \geqslant(1-\delta)\left|\int V_{1} d \mathbf{r}\right|
$$

(область и ее объем обозначаем одной и той же буквой). В совокупности (5.4) и (5.5) дают, как легко проверить, неравенство

$$
V_{0}(t, \Delta \mathbf{R} ; \nu) \int_{\Omega(\delta)} F_{1}^{(\mathrm{eq})}(\mathbf{r} \mid \mathbf{R} ; \nu) d \mathbf{r}+(1-\delta) \int V_{1}\left(t, \mathbf{R}, \mathbf{r} \mid \mathbf{R}_{0} ; \nu\right) d \mathbf{r} \geqslant 0 .
$$

Комбинируя его с первым из вириальных соотношений (4.7) (для $n=0$ ) и учитывая, что в рассматриваемом случае $\varkappa(\nu) \rightarrow 0$ и $F_{1}^{(\text {eq })}(\mathbf{r} \mid \mathbf{R} ; \nu) \leqslant 1$, после вполне очевидных рассуждений получаем

$$
\bar{\Omega}(t, \Delta \mathbf{R} ; \nu) V_{0}(t, \Delta \mathbf{R} ; \nu)+\frac{\partial V_{0}(t, \Delta \mathbf{R} ; \nu)}{\partial \nu} \geqslant 0,
$$

где величина $\bar{\Omega}(t, \Delta \mathbf{R} ; \nu)$ определена следующим образом:

$$
\bar{\Omega}(t, \Delta \mathbf{R} ; \nu)=\min _{0<\delta<1} \frac{\Omega(\delta, t, \Delta \mathbf{R} ; \nu)}{1-\delta} .
$$

Обсудим смысл этой величины. Во-первых, заметим, что $\Omega(\delta) \rightarrow 0$ при $\delta \rightarrow 1$ по определению области $\Omega(\delta, t, \Delta \mathbf{R} ; \nu)=\Omega(\delta)$. Во-вторых, если функция $V_{1}\left(t, \mathbf{R}, \mathbf{r} \mid \mathbf{R}_{0} ; \nu\right)$ в зависимости от $\mathbf{r}-\mathbf{R}$ знакопостоянна (при фиксированных прочих аргументах), то при $\delta \rightarrow 1$ эта область сжимается в исчезающе малую окрестность точки максимума $\left|V_{1}\right|$ (или в объединение таких окрестностей), так что можно написать

$$
\begin{aligned}
\left|\int_{\Omega(\delta)} V_{1} d \mathbf{r}-\int V_{1} d \mathbf{r}\right| & \rightarrow\left|\int V_{1} d \mathbf{r}\right|-\Omega(\delta) \max _{\mathbf{r}}\left|V_{1}\right|, \\
\Omega(\delta) \max _{\mathbf{r}}\left|V_{1}\right| & \rightarrow(1-\delta)\left|\int V_{1} d \mathbf{r}\right| .
\end{aligned}
$$

Следовательно,

$$
\bar{\Omega}(t, \Delta \mathbf{R} ; \nu) \leqslant \frac{1}{\max _{\mathbf{r}}\left|V_{1}\right|}\left|\int V_{1} d \mathbf{r}\right| .
$$

С другой стороны, вследствие (5.6) заведомо выполняется неравенство

$$
\bar{\Omega}(t, \Delta \mathbf{R} ; \nu) \geqslant \frac{1}{\max _{\mathbf{r}}\left|V_{1}\right|}\left|\int V_{1} d \mathbf{r}\right| .
$$

Отсюда (с учетом знакопостоянства) заключаем, что

$$
\bar{\Omega}(t, \Delta \mathbf{R} ; \nu)=\frac{1}{\max _{\mathbf{r}}\left|V_{1}\left(t, \mathbf{R}, \mathbf{r} \mid \mathbf{R}_{0} ; \nu\right)\right|} \int\left|V_{1}\left(t, \mathbf{R}, \mathbf{r} \mid \mathbf{R}_{0} ; \nu\right)\right| d \mathbf{r} .
$$


Это не что иное, как эффективный объем области в пространстве $\mathbf{r}-\mathbf{R}$, на которую распространяется двухчастичная корреляция. Допустим теперь, что предположение о знакопостоянстве $V_{1}\left(t, \mathbf{R}, \mathbf{r} \mid \mathbf{R}_{0} ; \nu\right)$ как функции $\mathbf{r}-\mathbf{R}$ не выполняется, и обозначим через $\mathcal{D}$ ту часть пространства $\mathbf{r}-\mathbf{R}$, где $V_{1}$ имеет доминирующий знак, т.е. совпадающий со знаком интеграла $\int V_{1} d \mathbf{r}$. Аналогичные рассуждения теперь дают

$$
\begin{aligned}
\frac{1}{\max _{\mathbf{r}}\left|V_{1}\right|}\left|\int V_{1} d \mathbf{r}\right| & \leqslant \bar{\Omega}(t, \Delta \mathbf{R} ; \nu) \leqslant \frac{1}{\max _{\mathbf{r} \in \mathcal{D}}\left|V_{1}\right|}\left|\int V_{1} d \mathbf{r}\right| \leqslant \\
& \leqslant \frac{1}{\max _{\mathbf{r} \in \mathcal{D}}\left|V_{1}\right|} \int_{\mathcal{D}}\left|V_{1}\right| d \mathbf{r} .
\end{aligned}
$$

Как видно, в данном случае можно сказать, что $\bar{\Omega}(t, \Delta \mathbf{R} ; \nu)$ не превышает эффективного объема, в котором существенна двухчастичная корреляция.

Далее сопоставим неравенство (5.8) с гипотезой о том, что асимптотика распределения перемещения БЧ имеет гауссову форму (5.2). Из (5.8) следует, что эта гипотеза может быть верной, только если

$$
\bar{\Omega}(t, \Delta \mathbf{R} ; \nu) \geqslant-\frac{\partial \ln D}{\partial \nu}\left(\frac{\Delta \mathbf{R}^{2}}{4 D t}-\frac{3}{2}\right)=\frac{1}{\nu}\left(\frac{\Delta \mathbf{R}^{2}}{4 D t}-\frac{3}{2}\right)
$$

(мы учли, что для газа $D \propto \nu^{-1}$ ). Иначе говоря гипотеза верна, если эффективный объем, занимаемый двухчастичной корреляцией, не ограничен сверху при изменении $t$ и $\Delta \mathbf{R}$. Напротив, если он ограничен и

$$
\nu \bar{\Omega}(t, \Delta \mathbf{R} ; \nu) \leqslant c_{1}=\text { const }
$$

то гауссова асимптотика (5.2) запрещается. Но неравенству (5.8) не противоречит, например, асимптотика вида

$$
V_{0}(t, \Delta \mathbf{R} ; \nu) \rightarrow \frac{1}{(4 \pi D t)^{3 / 2}} \Psi\left(\frac{\Delta \mathbf{R}^{2}}{4 D t}\right) \Theta\left(\frac{|\Delta \mathbf{R}|}{v_{0} t}\right),
$$

где функция $\Psi(z)$ должна удовлетворять неравенству

$$
z \frac{d \Psi(z)}{d z}+\alpha \Psi(z) \geqslant 0, \quad \alpha \equiv \frac{3}{2}+c_{1}\left(-\frac{\partial \ln D}{\partial \ln \nu}\right)^{-1}=\frac{3}{2}+c_{1} .
$$

Следовательно, функция $\Psi(z)$ не может убывать на бесконечности быстрее, чем по степенному закону: $\Psi(z \rightarrow \infty) \propto 1 / z^{\alpha}$. Формула (5.3) из работы [21] отвечает значению $c_{1}=\nu \bar{\Omega}=2$.

Таким образом, теория неизбежно приводит к статистическим корреляциям, имеющим неограниченную протяженность если не в пространстве, как в случае (5.12), то во времени, как в случае (5.13). Остается выяснить, какой из них ближе к точному решению уравнений ББГКИ. С физической точки зрения второй вариант несомненно предпочтительнее. Действительно, согласно уравнениям (2.17), источником корреляций между БЧ и атомами являются их столкновения. Столкновению соответствует такое расположение БЧ и атома, когда вектор $\rho=\mathbf{r}-\mathbf{R}$ лежит в "цилиндре столкновений", ориентированном параллельно относительной скорости частиц 
$\mathbf{u}=\mathbf{p} / m-\mathbf{P} / M$ и имеющем радиус $\approx r_{b}$. При этом частицы не должны отстоять друг от друга на расстояние, значительно превышающее $\Lambda=\min \left(\Lambda_{a}, \Lambda_{b}\right)$, так как иначе их столкновение не состоялось бы из-за встречи какой-нибудь из них с прочими частицами. Следовательно, двухчастичные статистические корреляции сосредоточены в основном в центре цилиндра столкновений, при $-\Lambda \lesssim \mathbf{u} /|\mathbf{u}| \cdot \rho \lesssim \Lambda$. Объем этой пространственной области не зависит от импульсов частиц, поэтому естественно принять его как оценку объема $\bar{\Omega}(t, \Delta \mathbf{R} ; \nu)$. При этом очевидно, что он не зависит от $t$ и $\Delta \mathbf{R}$. Таким образом, $\bar{\Omega} \approx 2 \Lambda_{b} \pi r_{b}^{2}=2 / \nu$ (если считать, как в [21], что БЧ - это один из атомов). В результате приходим к $(5.15)$ с $c_{1}=\nu \bar{\Omega} \approx 2$.

\section{6. ЗАКЛЮЧЕНИЕ}

Итак, задача о термодинамически равновесном блуждании пробной БЧ в газе или жидкости сформулирована на языке классической статистической механики в рамках канонического гиббсовского ансамбля начальных условий. Соответствующее уравнение Боголюбова для производящего функционала ФР рассмотрено в терминах КФ, которые выделяют статистические корреляции между текущим состоянием среды (газа или жидкости) и суммарным перемещением БЧ в течение всего времени ее наблюдения (исторические корреляции). Показано, что производящие функционалы равновесных $Ф Р$ и зависящих от времени КФ инвариантны относительно определенной непрерывной группы преобразований их аргументов, включая плотность среды (среднюю концентрацию атомов).

Найденная группа инвариантности порождает точные вириальные соотношения, которые связывают полные наборы КФ, взятые при различных значениях плотности. С помощью этих соотношений показано, что корреляции всех порядков играют одинаково важную роль независимо от величины газового параметра, и поэтому стандартное "больцмановское" приближение к кинетике БЧ, отвергающее корреляции третьего и высших порядков, некорректно даже в пределе разреженного газа (пределе Больцмана-Грэда). Соответственно присущая этому приближению гауссова асимптотика плотности вероятностного распределения перемещения БЧ вполне определенно запрещается вириальными соотношениями. Разрешаемая ими асимптотика обладает длинными степенными хвостами (обрезанными на расстоянии порядка баллистического пробега). Этот вывод согласуется с результатами приближенных решений уравнений ББГКИ в работах [7], [21]. Он означает, что все составные части траекторий БЧ статистически зависимы друг от друга. Поэтому траектории нельзя имитировать последовательностью независимых (в смысле теории вероятностей) случайных испытаний (выражаясь словами Эйнштейна, "Бог не играет в кости"). В этом смысле реальное молекулярное случайное блуждание, в отличие от блуждания в газе Лоренца (см. раздел 1), неэргодично.

Следует заметить, что результаты настоящей работы легко распространяются на термодинамически неравновесное случайное блуждание под действием сторонней силы (в этой связи см. [11], [15], [22]). Интересно также было бы обобщить рассмотренную группу инвариантности на задачи, где в центре внимания находятся не избранные частицы, а коллективные и гидродинамические переменные. 
Благодарности. Автор благодарен своим коллегам И. Краснюку и Ю. Медведеву за полезные обсуждения.

\section{Список литературы}

[1] Я. Г. Синай, УМН, 25:2(152) (1970), 141-192.

[2] Н.С. Крылов, Работы по обоснованию статистической физики, Изд. АН СССР, М.-Л., 1950.

[3] А.Н. Колмогоров, Основные понятия теории вероятностей, Наука, М., 1974.

[4] Г. А. Гальперин, А. Н. Земляков, Математические бильлрды, Наука, М., 1990.

[5] В.И. Арнольд, А. Авец, Эргодические проблемы классической механики, РХД, Ижевск, 1999.

[6] Г. Н. Бочков, Ю. Е. Кузовлев, УФН, 141:1 (1983), 151-176.

[7] Ю.Е. Кузовлев, ЖЭТФ, 94:12 (1988), 238.

[8] Н.Н. Боголюбов, Проблемы динамической теории в статистической физике, Гостехиздат, М.-Л., 1946.

[9] Д. Рюэль, Статистическая механика. Строгие результаты, Мир, М., 1971.

[10] В. П. Силин, Введение в кинетическую теорию газов, Изд. ФИ РАН, М., 1998.

[11] Yu. E. Kuzovlev, Molecular Brownian motion and falsity of the 'law of large numbers', http: www .ma . utexas . edu/mp_arc-bin/mpa?yn=07-309, 2007.

[12] Л. Д. Ландау, Е. М. Лифшиц, Теоретическая физика Т. 5. Статистическая физика. Часть 1, Наука, М., 1976.

[13] Е. М. Лифшиц, Л. П. Питаевский, Теоретическая физика Т. 10. Физическая кинетика, Наука, М., 1979.

[14] Yu. E. Kuzovlev, A truth about Brownian motion in gases and in general, arXiv: 0710.3831; Thermodynamic restrictions on statistics of molecular random walks, arXiv:0803.0301; Molecular random walks in a fluid and an invariance group of the Bogolyubov generating functional equation, arXiv: 0804.2023.

[15] Yu. E. Kuzovlev, Virial expansion of molecular Brownian motion versus tales of "statistical independency", arXiv: 0802.0288.

[16] Г. Н. Бочков, Ю.Е. Кузовлев, ЖЭТФ, 72 (1977), 238-247; 76 (1979), 1071; 79 (1980), 2239-2251.

[17] G. N. Bochkov, Yu. E. Kuzovlev, Phys. A, 106:3 (1981), 443-479.

[18] П. Резибуа, М. Де-Ленер, Кинетическая теория жидкостей и газов, Мир, М., 1980.

[19] H. van Beijeren, O. E. Lanford III, J. L. Lebowitz, H. Spohn, J. Stat. Phys., 22:2 (1980), $237-257$.

[20] Yu. E. Kuzovlev, On Brownian motion in ideal gas and related principles, arXiv: 0806.4157.

[21] Yu. E. Kuzovlev, On statistics and $1 / f$ noise of Brownian motion in Boltzmann-Grad gas and finite gas on torus. I. Infinite gas, arXiv: cond-mat/0609515.

[22] Yu.E. Kuzovlev, Kinetical theory beyond conventional approximations and $1 / f$-noise, arXiv: cond-mat/9903350.

[23] Ю. Е. Кузовлев, Г. Н. Бочков, Изв. вузов. Сер. радиофизика, 26:3 (1983), 310-317; 27:9 (1984), 1151-1157.

Поступила в редакцию 22.07.2008, после доработки 25.12.2008 